\title{
Epidemiology, diagnosis and treatment of the malignant pleural mesothelioma, a narrative review of literature
}

\author{
Sven Oliver Schumann ${ }^{1}$, Gregor Kocher ${ }^{2}$, Fabrizio Minervini $^{3}$ \\ ${ }^{1}$ Department of General Internal Medicine, Hospital Bülach, Bülach, Switzerland; ${ }^{2}$ Division of General Thoracic Surgery, Inselspital, Bern \\ University Hospital, University of Bern, Switzerland; ${ }^{3}$ Department of Thoracic Surgery, Lucerne Cantonal Hospital, Lucerne, Switzerland \\ Contributions: (I) Conception and design: All authors; (II) Administrative support: All authors; (III) Provision of study materials or patients: All \\ authors; (IV) Collection and assembly of data: SO Schumann; (V) Data analysis and interpretation: All authors; (VI) Manuscript writing: All authors; \\ (VII) Final approval of manuscript: All authors. \\ Correspondence to: Sven Oliver Schumann. Südweg 8, 8180 Bülach, Switzerland. Email: SvenOliverSchumann@web.de.
}

\begin{abstract}
The malignant pleural mesothelioma is a very aggressive tumor which is arising from mesothelial cells and is associated with asbestos exposure. It is a heterogeneous cancer that shows a complex pattern of molecular changes, including genetic, chromosomic, and epigenetic abnormalities. The malignant pleural mesothelioma is characterized by a silent and slow clinical progression with an average period of 2040 years from the asbestos exposure phase to the start of the symptoms. Unfortunately, to date, the therapeutic options are very limited, especially if the tumor is detected late. This narrative review provides an extended overview of the present evidence in the literature regarding the epidemiology, diagnostic pathways and treatment approaches of the malignant pleural mesothelioma. The treatment of mesothelioma has evolved slowly over the last 20 years not only from a surgical point of view but also radiotherapy, chemotherapy and immunotherapy play nowadays a key role. Several surgical strategies are available ranging from extrapleural pneumonectomy to cytoreductive surgery but a multidisciplinary approach seems to be mandatory because a single approach has not proved to date to be resolutive. New non-surgical treatment options appear to be promising but the results have to be taken in account with caution because clear evidence with high-quality studies is still lacking
\end{abstract}

Keywords: Malignant pleural mesothelioma (MPM); MARS, pleural disease

Submitted Aug 28, 2020. Accepted for publication Feb 21, 2021.

doi: $10.21037 /$ jtd-20-2761

View this article at: http://dx.doi.org/10.21037/jtd-20-2761

\section{Introduction}

Malignant pleural mesothelioma (MPM) is an uncommon but very aggressive intrathoracic carcinoma associated with a poor prognosis (1). In most cases the final cause of death is unclear (2). Eighty percent of patients (3) with MPM have at some point been directly or indirectly exposed to asbestos fibers (4). The MPM stems from mesothelial cells of the pleura, followed by an infestation of the lung, the diaphragm and the pericardium (1). Initially the onset of the disease is gradual with an estimated latency period of 20-40 years after the initial exposure (5). Due to its aggressive nature, MPM is often diagnosed in a late stage of disease and the remaining survival is significantly decreased. When untreated, less than $5 \%$ of patients survive 5 years, the average being 4-12 months post diagnosis depending on various factors such as age, histopathologic subtype, stage of disease, and other pre-existing comorbidities and therapies $(6,7)$. The use of asbestos is nowadays banned in 55 countries worldwide, including the United States, Western Europe and Australia (8). Despite the ban there is still no measurable decrease of MPM, with new cases still appearing due to the late onset of the disease. On the other hand, the use of asbestos in developing countries is still increasing $(8,9)$. Overall, it can also be assumed that there is a high number of unreported cases, especially in developing 
countries $(10,11)$. The World Health Organization (WHO) estimates that in 2004 approximately 107,000 people died from asbestos-related diseases like lung cancer, asbestosis and mesothelioma worldwide. It's also estimated that 12 times that amount of people (125 million) are exposed to asbestos fibers at work (12). Driscoll et al. showed in 2005 "The Global Burden of Disease Due to Occupational Carcinogens" that annually 43,000 people die from mesothelioma (13). The numbers suggest that every hour 5 to 10 people around the world die from mesothelioma or an asbestos-related disease.

One tragic example of mass exposure to asbestos in the recent past was 9/11. The World Trade Center collapsed, engulfing the streets of New York, thousands of its citizens, dousing them in dust full of asbestos fibers (14). The mass of the effects will show in the decades to come. Because of the late onset of this disease and the further use of asbestos fibers and currently not banned fibers, MPM will continue to be a challenge for healthcare systems worldwide, for decades to come (5). We present the following article in accordance with the Narrative Review reporting checklist (available at http://dx.doi.org/10.21037/jtd-20-2761).

\section{Methods}

This article is a hand searched narrative review of the literature. We searched PubMed for articles in English language on the following keywords alone or in combination: malignant pleural mesothelioma, treatment, surgery. Relevant papers and linked articles published between 1990 and July 2020 were included and screened.

\section{Epidemiology}

Asbestos started being used industrially in the 1880's (4). The use of asbestos became more popular in 1920. In the 1960 's there was an increase of MPM due to unknown danger, circulating asbestos in the times before, during and after World War II $(9,10)$. In industrialized countries the production and use of asbestos increased well into the $20^{\text {th }}$ century, where the use of 6 out of 400 potential carcinogen fibers was regulated and banned. The strict regulated fibers can be assigned to two subcategories:

- Serpentine class (chrysotile);

- Amphibole class (amosite, crocidolite, tremolite, actinolite and anthophyllite).

All 6 fibers are grouped under the collective term of "asbestos". The other fibers are yet to be regulated, although it seems that many of them could also be carcinogenic $(8,9)$. In the 1980 's it was assumed that only commercially used fibers could lead to harmful exposure, due to this, only the previously mentioned 6 fibers were banned (15). Asbestos was primarily used in the building industry due to its resilient nature and low-cost production. More than 3,000 different products with asbestos components were manufactured $(4,16)$. By outlawing asbestos in the European Union and other countries in 1999, world consumption dropped to an all-time low in the late 1990's. Simultaneously consumption increased in Asia, South America and Eastern Europe. Russia and China are currently two of the main producers and consumers of asbestos $(8,16)$. Since 2005 , the production and consumption of asbestos is fully banned in the European Union $(17,18)$. Allen et al. showed that the ban of asbestos did not have a negative long-term effect on the economy's growth, potentially reducing healthcare cost for the future. The careless handling of asbestos in the past is estimated to result in healthcare expenditures of US \$ 2.4-3.9 billion per year worldwide (16). Lin et al. proved with a comparison of data from 1960-1969 and 2000-2004 that the mortality rate of MPM was significant higher for men roughly 37.5 years after the time in which the production/use of asbestos reached its pinnacle (19).

\section{Pathophysiology}

Exposure to asbestos can occur during childhood, naturally or environmentally. If the patient is a carrier of an inherited germline mutation, men and women show nearly a $1: 1$ distribution with an early onset ( $<55$ years) of asbestos related disease and an increase of mesotheliomas $(8,9,20,21)$. Vivero et al. compared 36 young $[<35$ years, w $(47 \%)$ vs. m (53\%)] with 48 elderly patients [ $>35$ years, w $(13 \%) v s . \mathrm{m}(87 \%)]$. The histological transmission in both test groups was not significant. In older patients mostly a biphasic (40\%) or sarcomatoid (4\%) histology was found. However, in the younger study group most MPM had an epithelioid histology (78\%), biphasic (19\%) was the second most frequent histology and the least frequent histology was sarcomatoid (3\%). Previous radiation therapy was more common in younger patients, as their exposure to asbestos was more limited, than that of older patients. This finding suggests that a previous cancer history and radiotherapy could be a risk factor for developing MPM in young patients (21). Other studies suggest that an inherited BRCA1-associated protein 1 (BAP1) mutation is associated 
with an increased probability of MPM after exposure to asbestos, which is also described as gene $\times$ environment interaction [GxE] $(9,22)$. Carbone et al. reported that BAP 1 seems to have an important role in the development of mesothelioma. They analyzed two families in which a significantly increased mesothelioma rate occurred. In both families there was an inherited germline mutation of the BAP1 gene found, which seems to have dramatic influence on tumor growth (9).

Furthermore, in more than $60 \%$ of the cases a BAP1 mutation seems to be the current most mutated gene in patients with mesothelioma (23). Relating to this, Bononi et al. summarizes the important role in tumor growth of BAP1, in form of an influential tumor suppressor gene. They describe that descendants to whom the mutation is inherited, often develop at least one BAP1 associated tumor disease in their life, which is, in most cases, a malignant mesothelioma (24). Baumann et al. reported, even if in a small patient collective, that in $40 \%$ of cases MPM patients with a germline BAP1 mutation had more than one type of cancer, but that they also had a 2.3 -fold improved 5 -year survival rate, compared to patients without this mutation. Among other things, this could be because of an earlier diagnosis (25). A mutation in genes which repair damaged parts of the DNA can have devastating effects. Genes like BRCA2, MLH1, MLH3 and TP53 in a damaged form, often result in tumor growth. Recent studies show that oxygen radicals, which are caused by chronic inflammation, from the deposition of the asbestos fibers, could be the reason of tumor expansion (9). This inflammation could induce the activation of nuclear factor kappa-lightchain-enhancer of activated $\mathrm{B}$ cells $(\mathrm{NF}-\kappa \mathrm{B})$ and the phosphatidylinositol 3-kinase (PI3K) pathways inducing an uncontrolled growth of mesothelial cells.

\section{Histology}

Meyerhoff et al. showed 2015 in a review, which histological subgroup (sarcomatoid, biphasic and epitheloid) benefited from surgical therapy and which did not. They used data of the Surveillance, Epidemiology, and End Results (SEER) program, this is a program from the United States National Cancer Institute. They analyzed data only from patients with known histology from 2004-2010. Most patients were older than 70 years old, had a MPM on the right side of the chest, where male and fair skinned. Most patients had an epitheloid subtype [811 out of $1,183(69 \%)$ ], the second most common was the sarcomatoid subtype [224 out of 1,183 (19\%)] and least frequent was the biphasic subtype [148 out of 1,183 $(12 \%)]$. The median overall survival differed, patients with sarcomatoid histology died earlier (within 4 months), then the patients with biphasic histology (10 months) and patients with epitheloid histology (14 months) $(9,26)$. Compared to patients, which had a surgical treatment showed a median survival of 4 months with sarcomatoid histology, 12 months with biphasic histology and 19 months in the group of patients with epitheloid histology. In the group of Patients with no surgical treatment median survival was 3 months in patients with sarcomatoid histology, 10 months with epitheloid histology and 12 months with biphasic histology. Although the number of cases was not the same for all groups, results suggest that patients with epitheloid histology could possible benefit the most from surgical treatment, and patients with biphasic histology may could be at a disadvantage. The authors also reported data from the IASLC, of patients which underwent an operation, this data indicates a median survival of 8 (sarcomatoid histology), 13 (biphasic histology) and 19 (epitheloid histology) months (26). Brims et al. reported of a median survival of 7.5 months in patients with sarcomatoid histology, 9.1 months in Patients with biphasic histology and 14.8 months in patients with epithelioid histology (27). Recent studies show a subgrouping of the individual histologies (C1 and C2). With different molecular profiles, gene modifications and survival outcomes (28).

\section{Diagnostic}

The manifestation of the MPM is displayed with unspecific symptoms such as shortness of breath (caused by pleural effusion), chest pain, fatigue and a dry cough. However, the typical B symptoms can also appear. The next step, after an accurate medical history and physical examination, should be an X-ray and/or CT of the thorax, or a noninvasive ultrasound of the pleura. Pleural plaques on X-ray were evident in more than $80 \%$ of cases from patients with asbestos exposure and MPM. It is common after the first line of diagnostic, to scrutinize the pleural effusion by a cyto-/histological examination. Because of low sensitivity, fine needle biopsy is commonly not recommended (29). For Carbone et al., pleural effusion is the first material to be obtained, here the experience of the examiner is crucial, because the sensitivity can vary from $<5$ to $>90 \%$. A Thoracoscopic biopsy is a possible means to drastically 
improve accuracy. After the diagnosis of MPM, a second assessment should be carried out by an experienced specialist, but even when using highly sensitive and specific methods such as immunohistochemistry (IHC), a misdiagnosis cannot be ruled out (9). As stated by the ERS/ ESTS/EACTS/ESTRO task force, VATS pleural biopsies (similar to the medical thoracoscopy) carry a sensitivity of $95 \%$, specificity of $100 \%$ and negative predictive value of $94 \%$ offering the possibility to secure a pathological diagnosis, assess the pleural cavity for staging purposes and perform a talc poudrage (30). There are three known histological subtypes of MPM: epithelioid, sarcomatoid and biphasic. According to the recommendations of the American Society of Clinical Oncology, an individual patient-specific diagnosis should be made. To carefully differentiate an MPM from a carcinoma or sarcoma, a cytological and immunohistochemical examination is crucial because a misdiagnosis would have far-reaching consequences for a patient. If symptomatic pleural effusions are present, ultrasound-guided thoracentesis with subsequent cytopathological examination should be performed as one of the first diagnostic steps; however, owing to its low sensitivity, a cytopathological examination of the pleural effusions can correctly diagnose only less than one-third of MPM cases (31) [it is most sensitive in patients with epithelioid histology (32)]. Furthermore, a drainage of the pleural effusions helps to reduce symptoms such as chest discomfort or dyspnea. With the requirement of making as few incisions as possible in the area of the subsequent operation side in order to prevent parietal tumor spreading, if these procedures are not possible, a fine needle biopsy is recommended despite its low sensitivity. Thoracoscopic biopsy, open pleural biopsy or fine needle biopsy helps to conclude the clinical staging to determine the histology. Cases should be subdivided according to histological differentiation, because for example, the SEER database indicates that among patients who underwent surgery, patients with an epithelial subtype had a median survival of 19 months, whereas patients with biphasic and sarcomatoid subtypes had a median survival of 12 and 4 months, respectively. IHC could be used as a tool for differentiating between MPM and other carcinomas. For example, in MPM, positive immunohistochemical results are observed with Wilms tumor protein 1, keratins 5/6, podoplanin and calretinin. However, patients with MPM with a loss of BRCA1-associated protein (BAP1) seem to have a better prognosis (33). On the other hand, there are negative immunohistochemical results in patients with
MPM, for example, epithelial cell adhesion molecule, carcinoembryonic antigen, blood group 8 , napsin $\mathrm{A}$, thyroid transcription factor 1 and Claudin 4. Another approach to differentiate between MPM and other carcinomas is to test for specific markers for differential diagnosis $(34,35)$. Furthermore, fluorescent in situ hybridization could indicate a genomic loss of p16/CDKN2A locus at 9p21 in patients with MPM (36).

\section{Staging}

Staging-scores and systems were numerous in the last decades. Due to poor data availability, the International Association for the Study of Lung Cancer (IASLC) has set itself the task to build a proofed and validated database. The current 2nd Edition by IASLC 2016, includes data from 1995-06/2013. For the 8th Edition of the Lung Cancer Staging System, by the IASLC, the data of 2,460 out of 3,519 Patients with MPM from 29 Institutes, from 4 different continents were analyzed. The collected data serve as the basis for the changes in the staging and TNM categories of MPM, which are presented in the 8th Edition of the Lung Cancer Staging System, by the IASLC. As with other tumor diseases, the MPM is classified according to a TNM classification, which then results in a stage classification from Stage IA - Stage IV (37). Owing to the small number of cases, it is difficult to create a staging system that is widely accepted. The first staging step should be a CT scan of the thorax and the upper abdomen with IV contrast medium in order to identify baseline characteristics. If the clinical radiologist is familiar with modified Response Evaluation Criteria in Solid Tumor (RECIST) and has the expertise available, T-staging can be performed by measuring according to modified RECIST for mesothelioma (38-40). Data of measurements of tumor thickness (TT) can be used to demonstrate the response of the ongoing therapy. To avoid inter-observer variability, a single radiologist or a defined team of radiologists should perform the analysis for all patients $(40,41)$. In patients who are candidate for surgery a FDG PET/CT should be performed because, in approximately $10 \%$ of cases, it could identify metastatic spread, which is not detected by CT scan. FDG PET/CT helps to distinguish malignant differentiations from benign forms $(42,43)$. Furthermore, the extent of FDG absorption is prognostic for outcome. If CT scan of the thorax/abdomen or PET/CT shows structures suspected to be metastatic, a CT scan of the abdomen with IV and oral contrast media is recommended. 
However, cerebral metastases are rare and should therefore be explored only in the presence of symptoms. Because symptoms of MPM such as dyspnea and shortness of breath, are promptly clarified, there is often no distant metastasis at the time of diagnosis. However, the ipsilateral chest wall, contralateral pleura and peritoneum may be affected at the time of diagnosis. Particularly, if the carcinoma has invaded into the mediastinum, diaphragm, thorax or other areas, a CT scan is often not as accurate as required. In such cases, if the patient is still a candidate for surgery, an MRI with IV contrast medium should be performed to reveal infiltrative structures in the soft tissue; however, the MRI is more expensive and needs more expertise of the observer, than a CT scan (44). In addition to radiological examinations, invasive staging techniques are required to confirm the radiological diagnosis with a subsequent cytopathological analyses. For example, EBUS-guided fine-needle aspiration has a higher specificity and sensitivity in nodal variations than cervical mediastinoscopy (45). Thoracoscopy provides crucial information's about histologic subtype and molecular expression but, in cases of radiologically suspected mediastinal lymph nodes metastasis or contralateral carcinoma, it could play a key role in the decision-making process. Moreover, laparoscopy can be used as a staging method to supplement the data from radiological imaging, especially in cases in which the carcinoma extends through the diaphragm [T4] or if intraabdominal manifestation is expected $(46,47)$. According to the IASLC, an ipsilateral involvement of intrathoracic lymph nodes indicates N1 disease. Because most lymph nodes are difficult to access, the accuracy of clinical diagnosis is limited. T-staging can be challenging, especially in the early stages of the disease, because there is often a discrepancy between radiological diagnosis and pathological staging. In radiological examinations, it is often difficult to measure the exact depth and spread in the pleural space, indicating that patients with clinical stage I/II cancer may need intraoperative upstaging. On the contrary, usually only a CT scan of the thorax is needed for patients who are not candidate for surgery but are suitable for chemotherapy alone and/or radiotherapy and/or immunotherapy in order to evaluate the response to treatment.

\section{Prognostic factors}

Different scoring systems can be found in the international Literature even if they have been extrapolated from retrospective studies. The greatest advantages given by prognostic scores is the possible use of an individual case based, patient-oriented therapy, which could improve outcomes (29). Brims et al., reported in their publication, with a collective of 482 patients including patients with MPM from 2005-2010 and from 2011-2014, weight loss (classified as significant by a medical professional) as one of the most important predictive factors. Patients who did not lose weight, had a hemoglobin $>153 \mathrm{~g} / \mathrm{L}$ and a serum albumin $>43 \mathrm{~g} / \mathrm{L}$, had the best survival rate at 18 months and a median survival of 34 months. Patients with sarcomatoid histology and recorded weight loss only had a median survival of 7.5 months (27). Opitz et al., presented their Multimodality Prognostic Score (MMPS) which names 3 prognostic factors pre-chemotherapy: volume of the MPM $(>500 \mathrm{~mL}), \mathrm{CRP}>30 \mathrm{mg} / \mathrm{L}$ and a confirmed histological non-epithelioid subtype. A progressive disease of MPM as a negative prognostic post-chemotherapy factor in their score is also mentioned. The analyses showed a significantly shorter overall survival of patients, with a score greater than 3 (48). Sugarbaker et al., analyzed other prognostic factors in 2010: female gender, age of 56 years or younger, histological secured epithelial tumor, normal blood parameters (hemoglobin, white blood cells and platelets) before operation (1). In addition, there are prognostic factors mentioned in other publications like the restriction of the tumor to one half of the thorax, missing evidence of histological lymph node involvement and a slight cancer spread (29). de Perrot et al., showed in their data analysis the possible role of the TT on the survival rate. The patients underwent an extra pleural pneumonectomy (EPP) after a high-dose radiation of the affected thorax. In their data analysis, they found that both tumor volume $\left(<500 \mathrm{~cm}^{3}\right.$ + epitheloid histology) and diaphragmatic TT $(<2 \mathrm{~cm})$ had an impact on median and disease-free survival (49).

\section{Surgical treatment}

The actual importance of surgical treatment is unclear, it seems that only some patients benefit from an aggressive therapy, but it appears reasonable to perform mesothelioma surgery only in MPM high volume centres, due to the perioperative risks and different experiences (50). Sugarbaker et al., showed in 2010 that a multimodal therapy of patients with MPM, with a surgery-based therapy (EPP) was associated with a long-term survival rate over 3 years in $18 \%$ of the patients (117 of 636 ) (1). The role of surgical treatment was shown by an end result study of 14,228 patients published in 2015, which showed that a surgical 
treatment alone, was significantly associated with survival. Patients who undergo surgery lived significantly longer if compared with patients who undergo radiotherapy or do not receive any therapy (51). An EPP is an extended operation, which includes an en bloc resection of the entire lung, the visceral and parietal pleura, the pericardium and diaphragm. Furthermore, it is necessary to resect lymph nodes, which are found along the intercostal structure in the mammary chain, in addition a radical mediastinal lymphadenectomy is needed. To prevent herniation, it is of upmost importance to reconstruct the pericardium and diaphragm with patches. Some surgeons believe that the maximal macroscopic cytoreduction (MCR) of the tumor should be the goal of the surgical treatment as in other oncological treatment settings (29). Brims et al., reported in their publication about a small group of 12 patients who underwent EPP with a median survival of 19.5 months (5.5-33.5) (27). It also seems that patients could benefit from a combination of therapy options. In addition to the MCR due to EPP, therapy of micro metastasis is paramount. This result is only reachable with a multimodal therapy of the MPM (52). With advanced operative techniques, the perioperative care is very important. Invasive hemodynamic monitoring and a selective use of bronchoscopy is common. Furthermore, a protective lung ventilation is needed to prevent acute respiratory distress syndrome. An adequate perioperative management also includes an intensive care unit team with understanding of the special needs of patients after pneumonectomy (29). Sugarbaker et al. reported in a study with 328 patients that the most common complication after extrapleural pneumonectomy is reversible atrial fibrillation, which was seen in 145 out of 328 patients, this shows the importance of a prophylaxis of atrial fibrillation which was not part of this study. The most common postoperative complications associated with death are pulmonary embolism, ARDS and myocardial infarction (53). Opitz et al. name heart failure, pulmonary embolus, septic multiple organ dysfunction syndrome (MODS), pneumonia and gastric necrosis as deathassociated causes after EPP (48). The ERS/ESTS/EACTS/ ESTRO task force which included several experts in MPM research and management elaborated an algorithm where the central point is: is the patient suitable for a macroscopic surgical resection (MCR) in the context of a multimodality treatment including chemotherapy and radiotherapy. If the patient is to frail for MCR, a partial resection with a palliative surgical approach is possible. The MPM (MesoVATS) trial was published in Lancet in 2014, it was designed as a multicenter, controlled and computer randomized open label trial. It compares the costs and the overall survival of patients after different therapy options. In this trial a talc pleurodesis was compared with a videoassisted thorascopic partial pleurectomy (VAT-PP). 196 Patients were recruited from 10/2003-01/2012, 175 of them had a confirmed pleural mesothelioma. In the end out of 175 patients, 73 patients were treated with talc pleurodesis and 78 with VAT-PP. The cohort of the talc pleurodesis had a better overall survival after the first year with $57 \%$. In the VAT-PP group the 1 -year survival was merely $52 \%$. Furthermore, the talc pleurodesis cohort had less surgical complications (14\%) than the VAT-PP cohort (31\%). Besides that, VAT-PP treatment was 5,000\$ more expensive than the comparable costs of a talc pleurodesis. However, the study showed that the quality of life of those from the VAT-PP cohort was better after 6 months, than in patients who underwent a talc-pleurodesis. In synopsis of all results the authors does not recommend VAT-PP for treatment in patients with malignant pleural mesothelioma. Talc pleurodesis is considered a better option due to a lower complication rate and a shorter hospitalization (54). If the patient is fit enough MCR should be performed. The MARS trail tries to answer the question which surgical technique has the best outcome. The Mesothelioma and Radical Surgery (MARS) trial, published in 2011, compares the survival of patients who undergo a triple therapy (a combination of chemotherapy plus EPP plus radiotherapy) with patients who undergo chemotherapy and no EPP. The MARS trial was a multicenter randomized controlled trial, which showed a median survival of the EPP cohort of 14.4 months (5.3-18.7) and a median survival of the no EPP cohort of 19.5 months (13.4-lifetime exceed observation time). In this study patients undergo a triple therapy; 112 patients were included from 10/2005 to 11/2008 and all of them had a platinum-based chemotherapy. Less than half of patients [50] were computer based randomized, 24 should get an EPP, in the end 16 patients underwent an EPP and 8 of them got radiotherapy. Two patients died in less than 30 days and one patient died during hospitalization. Twenty-six of fifty patients had no EPP. The quality of life analysis showed no significant difference in either cohort, patients of the EPP cohort however had a worse outcome than patients of the no EPP cohort. In summary, the authors concluded that EPP is not a recommended treatment for the next trial, it seems that a triple therapy does not offer advantages, rather it may impair patients (55). The results of this study show one time more, that a 
randomized trial seems to be difficult or not to be workable and that selection bias are more common in trails with small patient collectives. Due to results from the previous MARS trial, there is currently a new trial, the MARS 2 study which compares standard chemotherapy (combination of pemetrexed and cisplatin) alone with surgical treatment [extended pleurectomy decortication (EPD)] including the standard chemotherapy, designed as a randomized trial. The aim of an EPD is the removal of all macroscopic visible tumor mass, a decortication of the lung and the pleura. If there is tumor mass at the diaphragm and/or pericardium it will also be removed. We expect results from the current study in the next few years (56). A long-term Meta-Analysis, which spanned a period of almost 30 years showed, that the median overall survival in the pleurectomy/decortication (P/D) group and the 30-day mortality in the EPP group is significantly higher (57-59). In addition, there were more perioperative complications in the group of EPP patients $(57,58,60)$, besides that patients after P/D have a higher forced vital capacity (FVC), forced expiratory volume (FEV1) and quality of life (QoL) (61). Even if the results seem devastating, there is still a place for EPP today. Trials show also acceptable mortality and morbidity rates for a selected group of patients in the context of multimodal therapy at high-volume centres (62-64). The MesoTRAP trial was published in 2018, it's a randomized controlled, pilot multicenter feasibility study. Which compares the treatment of video-assisted thoracoscopic partial pleurectomy decortication (VAT-PD) with an indwelling pleural catheter (IPC) in patients with pleural effusion and significant trapped lung by confirmed MPM. Included in the trial were only patients eligible for both treatment options. In the advanced stage of the disease many are affected by and suffer serve respiratory symptoms, therefore their aim is to specifically explore treatment of patients with trapped lung within MPM. The Aim of the feasibility study was to randomize and recruit 38 patients within 18 months, if these patients aren't impaired by trial interventions, they would like to set up a phase III trial. The main goal of the feasibility study is to determine the standard deviation (SD) of VAS scores for dyspnea, over the entire observation period. The main subordinate goals are the exploration of the subjective quality of life during different phases of the study and the survival rates of the patients. Results from current studies are expected in a few years (65). A study by Opitz et al., examined the approach of a fibrin-cisplatin mixture, for intracavity application after (extended) pleurectomy/decortication. Twelve patients were included in their study, 8 of whom had previously received a systemic cisplatin and pemetrexed based chemotherapy. Out of the 12 patients there were 5 subgroups with 4 different concentrations of fibrin-cisplatin mixture. Initially, all patients underwent an (extended) pleurectomy/ decortication. Thereafter, all resection surfaces were sprayed with a fibrin-cisplatin mixture under video assistance. After surgery and the spraying of the fibrincisplatin mixture, there was no other treatment. Compared with other trials, which use hyperthermia, which was not used in this trial, and intracavitary cisplatin perfusion, the renal toxicity was reduced. No fatalities were observed in the first 90 days of observation. Furthermore, the trial showed an overall survival of 10-31 months with a median of 21 months. However, the available data show that an intracavitary therapy with a mixture of cisplatin and fibrin is safe up to the maximum concentration of $44 \mathrm{mg} / \mathrm{m}^{2}$, which was tested in this trial. Due to the limited test group, the results are of limited use. For this reason, a phase II study is currently being run to generate more data and to confirm the effectiveness of this new therapy option (66).

\section{Chemotherapy}

A multimodal therapy regime also can include a chemotherapy, besides that chemotherapy could be an option in macroscopic or radiological not respectable MPM. Different combinations of chemotherapy are used; Vogelzang et al., reported that a combination of cisplatin and pemetrexed can help to improve the overall survival, progression-free survival and response rate compared with a single-agent therapy with cisplatin (67). The South West Area Mesothelioma and Pemetrexed (SWAMP) trial compared patients who received a combination of cisplatin and pemetrexed with patients who chose best supportive care (BSC), demonstrating that patients treated with the combination therapy had fewer symptoms and improved HR QoL at 16 weeks (68). In the Mesothelioma Avastin Cisplatin Pemetrexed Study (MAPS), triple therapy (standard pemetrexed/cisplatin + bevacizumab) showed improved overall survival and progression-free survival (69). However, because of insufficient data, there is no clear recommendation; it may improve survival in certain groups of patients without contraindications to bevacizumab. In patients responding to therapy or with stable disease, pemetrexed-based chemotherapy should be performed with pauses after 4-6 cycles. A multicenter trial showed that patients treated with a combination therapy of EPP, 
adjuvant hemithoracic radiation therapy and neoadjuvant chemotherapy with platinum/pemetrexed had a 2-year overall survival of approximately $61 \%$ with a median survival of 29.1 months (70). Another trial showed that combination therapy with raltitrexed/cisplatin also improved the overall survival of patients compared with treatment with cisplatin alone $(71,72)$. In patients with unresectable MPM who cannot tolerate cisplatin, it is possible to substitute cisplatin by carboplatin as data suggest a similar effectiveness. A Chemotherapy can significantly reduce symptoms such as pain and dyspnea, thus improving the quality of life (QoL). In a previous study, in patients with a $\leq 2$ performance status (PS), systemic combination chemotherapy with platinum plus pemetrexed, with vitamin (B12) and folic acid supplementation to reduce toxicity, was used as first-line therapy, which led to nausea (14.6\%), leukopenia (17.7\%) and grade $3 / 4$ neutropenia (27.9\%). Compared with a cohort treated with single-agent therapy with cisplatin, the cohort treated with combination therapy showed a higher response rate $(41.3 \%$ vs. $16.7 \% ; \mathrm{P}<0.001)$, median overall survival (12.1 vs. 9.3 months; $\mathrm{P}=0.020)$ and progression-free survival (5.7 vs. 3.7 months; $\mathrm{P}=0.001$ ). The median number of cycles of the combination therapy was six (67). Other therapy options can be offered as part of clinical studies. In the case of patient groups with a worse performance status ( $P S \geq 2$ ), single-agent chemotherapy-for example, with pemetrexed, vinorelbine or gemcitabine-is feasible. Patients who are not eligible for therapy should receive palliative care. If the PS of the patient is $\geq 3$, palliative care is indicated. For patients who reach disease control with pemetrexed-based chemotherapy as first-line therapy and need second-line therapy, pemetrexed-based chemotherapy is an option $(73,74)$. Ideally, these therapies should be given in clinical trials. In all other cases, vinorelbine treatment may be used (75). In certain cases, with epithelial histology and asymptomatic patients, an observational trial with close monitoring can be carried out before starting therapy. However, O'Brien et al., showed that early chemotherapy helps to extend survival and increase the period of symptom control (76).

\section{Radiation therapy}

In special cases, adjuvant/neoadjuvant radiation therapy can be offered to patients who are planned to undergo EPP. Ideally, this therapy should be carried out in trials at experienced centers $(77,78)$. The Intensity modulated pleural radiation therapy (IMPRINT)-trail showed an effect of radiotherapy who underwent a multimodal Therapy with chemotherapy and P/D (Phase II Study of Hemithoracic Intensity-Modulated). Especially patients with a respectable epithelioid MPM can benefit from an accelerated hemithoracic intensity modulated radiation therapy (IMRT) followed by an EPP (Accelerated hemithoracic radiation followed by extrapleural).Radiation techniques such as IMRT or three-dimensional (3D) conformal radiation therapy (CRT) can help to precise and control the radiation dose $(79,80)$. Clinical trials have reported dissimilar results; Rusch et al., reported a local control of up to $97 \%$ (78), whereas other trials reported a local control of up to $40-71 \%$, with a 2 -year overall survival of up to $18-57 \%(64,81-83)$. Side effects of radiation therapy include radiation-related lung damage in particular, which often affects the contralateral lung (84); reduced toxicity was observed, by decreasing radiation doses in combination with rising experience $(80,85)$. Patients who undergo a surgical procedure such as lung-sparing cytoreductive surgery (P/D or extended $\mathrm{P} / \mathrm{D})$ should not be given neoadjuvant radiation therapy owing to the high toxicity. However, adjuvant intensity-modulated radiation therapy in clinical trials at experienced centers is feasible in certain cases. New techniques such as IMRT make it possible to reduce the toxicity for untreated tissues through targeted use of radiation. Rosenzweig et al. reported that the risk of pneumonitis grade 3 or higher is approximately $20 \%$ (86). The larger the irradiated area ( $\geq 5 \mathrm{~Gy})$, the greater the risk of radiation damage to the lungs with the development of pneumonitis (87). Taking into account the standard dosimetric guidelines for organs, adjuvant or neoadjuvant hemithoracic radiation therapy with techniques such as IMRT, 3D CRT or proton therapy may be offered at specialized centers and/or in clinical trials. Preventing implantation metastasis with prophylactic irradiation is not recommended. There is no significant effect of survival or quality of life after prophylactic radiotherapy, in patients with MPM after a pleural intervention $(88,89)$. On the other hand, Boutin et al. showed with a small collective of patients that there were more subcutaneous nodules in the patients who did not undergo a prophylactic radiotherapy (90). However, for patients with implantation metastasis, as observed in histological examination, adjuvant radiation therapy should be offered. On the other hand, small trials did not show statistically significant results. The proponents of this therapy option cite a study by Boutin et al., which reported that patients who received radiotherapy (21 Gy in three consecutive fractions) together 
with thoracoscopic intervention had no implantation metastasis 10-15 days post intervention; without radiation, metastasis was observed in $40 \%$ of the cases (90). If radiation therapy is used, electrons are effective for superficial targets and implantation metastasis. In cases of local asymptomatic recurrence, patient-adapted therapy should be used. A decision should be made by a multidisciplinary team; all therapy options should be considered, and the best option should be offered to the patient. Radiation therapy can help to achieve local control, for example, with IMRT at centers of excellence. Radiation therapy (2D, 3D, IMRT, or electron) can be used in palliative care to reduce symptoms. Standard procedures used for other diseases can be used, for example, 3 Gy $\times 10$ fractions, 4 Gy $\times 5$ fractions or 8 Gy $\times 1$ fractions. In patients who undergo a palliative care, long-term toxicity as with stereotactic body radiation therapy lose importance (91-93). Data from the Symptom Study of Radiotherapy in Mesothelioma (SYSTEMS-1) showed optimal results for pain reduction in $47 \%$ of patients with a total dose of 20 Gy in five fractions (4 Gy x 5 fractions) (93).

\section{Conclusions}

Although therapies are improving and mortality rates are decreasing "the most efficient way to eliminate asbestosrelated diseases is to stop using all types of asbestos." Statement WHO 2014 (94). Desirable would also be if further large-scale studies on the treatment options of the MPM were carried out. In the future the histology and genotype should be a main focal point. Furthermore, patients with MPM should, if possible, be treated as part of study protocols at high-volume centers in order to collect further data for the future. If that's not possible data of the patients should be collected and presented in the context of smaller studies or case reports. For the best possible data collection and best prognosis of the patient, the therapy of patients with MPM should be discussed by an interdisciplinary team of pathologist, oncologists, radiotherapists and surgeons at a medical center with special knowledge or as a part of a study. Histological differentiation should be part of a therapy concept of MPM, as shown some histological subtypes affect the outcome of the therapy.

\section{Discussion}

This article is a narrative review. Therefore, a certain subjectivity in choice of studies included is likely. In narrative reviews like this one, the large number of sources and studies which are cited accepts that there is a complexity of interactions. The identification and integration of those could be difficult.

\section{Acknowledgments}

Funding: None.

\section{Footnote}

Reporting Checklist: The authors have completed the Narrative Review reporting checklist. Available at http:// dx.doi.org/10.21037/jtd-20-2761

Peer Review File: Available at http://dx.doi.org/10.21037/jtd20-2761

Conflicts of Interest: All authors have completed the ICMJE uniform disclosure form (available at http://dx.doi. org/10.21037/jtd-20-2761). The authors have no conflicts of interest to declare.

Ethical Statement: The authors are accountable for all aspects of the work in ensuring that questions related to the accuracy or integrity of any part of the work are appropriately investigated and resolved.

Open Access Statement: This is an Open Access article distributed in accordance with the Creative Commons Attribution-NonCommercial-NoDerivs 4.0 International License (CC BY-NC-ND 4.0), which permits the noncommercial replication and distribution of the article with the strict proviso that no changes or edits are made and the original work is properly cited (including links to both the formal publication through the relevant DOI and the license). See: https://creativecommons.org/licenses/by-nc-nd/4.0/.

\section{References}

1. Sugarbaker DJ, Wolf AS, Chirieac LR, et al. Clinical and pathological features of three-year survivors of malignant pleural mesothelioma following extrapleural pneumonectomy. Eur J Cardiothorac Surg 2011;40:298-303.

2. Finn RS, Brims FJH, Gandhi A, et al. Postmortem findings of malignant pleural mesothelioma: a two-center study of 318 patients. Chest 2012;142:1267-73. 
3. Tossavainen A. Asbestos, asbestosis, and cancer: the Helsinki criteria for diagnosis and attribution. Scand J Work Environ Health 1997;23:311-6.

4. National Toxicology Program, Department of Health and Human Services. Report on Carcinogens, Fourteenth Edition Asbestos, CAS No. 1332-21-4. Available online: https://ntp.niehs.nih.gov/ntp/roc/content/profiles/ asbestos.pdf

5. Scherpereel A, Astoul P, Baas P, et al. Guidelines of the European Respiratory Society and the European Society of Thoracic Surgeons for the management of malignant pleural mesothelioma. Eur Respir J 2010;35:479-95.

6. van Meerbeeck JP, Scherpereel A, Surmont VF, et al. Malignant pleural mesothelioma: the standard of care and challenges for future management. Crit Rev Oncol Hematol 2011;78:92-111.

7. Jaklitsch MT, Grondin SC, Sugarbaker DJ. Treatment of malignant mesothelioma. World J Surg 2001;25:210-7.

8. Stayner L, Welch LS, Lemen R. The worldwide pandemic of asbestos-related diseases. Annu Rev Public Health 2013;34:205-16.

9. Carbone M, Adusumilli PS, Alexander HR, et al. Mesothelioma: Scientific clues for prevention, diagnosis, and therapy. CA Cancer J Clin 2019;69:402-29.

10. Park E-K, Takahashi K, Hoshuyama T, et al. Global magnitude of reported and unreported mesothelioma. Environ Health Perspect 2011;119:514-8.

11. Delgermaa V, Takahashi K, Park EK, et al. Global mesothelioma deaths reported to the World Health Organization between 1994 and 2008. Bull World Health Organ 2011;89:716-24, 724A-724C.

12. World Health Organization. International Programme on Chemical Safety / Asbestos. (2017, November 27). Available online: https://www.who.int/ipcs/assessment/ public_health/asbestos/en/

13. Driscoll T, Nelson DI, Steenland K, et al. The global burden of disease due to occupational carcinogens. Am J Ind Med 2005;48:419-31.

14. Lorber M, Gibb H, Grant L, et al. Assessment of inhalation exposures and potential health risks to the general population that resulted from the collapse of the World Trade Center towers. Risk Anal 2007;27:1203-21.

15. Baumann F, Ambrosi JP, Carbone M. Asbestos is not just asbestos: an unrecognised health hazard. Lancet Oncol 2013;14:576-8.

16. Allen LP, Baez J, Stern MEC, et al. Trends and the Economic Effect of Asbestos Bans and Decline in Asbestos Consumption and Production Worldwide. Int J Environ
Res Public Health 2018;15:531.

17. WHO Regional Office for Europe. The Human and Financial Burden of Asbestos in the WHO European Region: Meeting Report 5-6 November 2012, Bonn, Germany. Available online: http://www.euro.who.int/ data/assets/pdf_file/0003/194133/RB-Asbestos-MtgReport-Bonn-2012.pdf?ua=1

18. European Union, COMMISSION DIRECTIVE 1999/77/EC of 26 July 1999 adapting to technical progress for the sixth time Annex I to Council Directive 76/769/ EEC on the approximation of the laws, regulations and administrative provisions of the Member States relating to restrictions on the marketing and use of certain dangerous substances and preparations (asbestos).: Official Journal of the European Communities 6.8.1999. Available online: https://eur-lex.europa.eu/LexUriServ/LexUriServ.do?uri= OJ:L:1999:207:0018:0020:EN:PDF

19. Lin RT, Takahashi K, Karjalainen A, et al. Ecological association between asbestos-related diseases and historical asbestos consumption: an international analysis. Lancet 2007;369:844-9.

20. Reid A, Berry G, Klerk N de, et al. Age and sex differences in malignant mesothelioma after residential exposure to blue asbestos (crocidolite). Chest 2007;131:376-82.

21. Vivero M, Bueno R, Chirieac LR. Clinicopathologic and genetic characteristics of young patients with pleural diffuse malignant mesothelioma. Mod Pathol 2018;31:122-31.

22. Carbone M, Kanodia S, Chao A, et al. Consensus Report of the 2015 Weinman International Conference on Mesothelioma. J Thorac Oncol 2016;11:1246-62.

23. Nasu M, Emi M, Pastorino S, et al. High Incidence of Somatic BAP1 alterations in sporadic malignant mesothelioma. J Thorac Oncol 2015;10:565-76.

24. Bononi A, Giorgi C, Patergnani S, et al. BAP1 regulates IP3R3-mediated $\mathrm{Ca}(2+)$ flux to mitochondria suppressing cell transformation. Nature 2017;546:549-53.

25. Baumann F, Flores E, Napolitano A, et al. Mesothelioma patients with germline BAP1 mutations have 7-fold improved long-term survival. Carcinogenesis 2015;36:76-81.

26. Meyerhoff RR, Yang CFJ, Speicher PJ, et al. Impact of mesothelioma histologic subtype on outcomes in the Surveillance, Epidemiology, and End Results database. J Surg Res 2015;196:23-32.

27. Brims FJH, Meniawy TM, Duffus I, et al. A Novel Clinical Prediction Model for Prognosis in Malignant Pleural Mesothelioma Using Decision Tree Analysis. J Thorac 
Oncol 2016;11:573-82.

28. Reyniès A de, Jaurand MC, Renier A, et al. Molecular classification of malignant pleural mesothelioma: identification of a poor prognosis subgroup linked to the epithelial-to-mesenchymal transition. Clin Cancer Res 2014;20:1323-34.

29. Bueno R, Opitz I. Surgery in Malignant Pleural Mesothelioma. J Thorac Oncol 2018;13:1638-54.

30. Scherpereel A, Opitz I, Berghmans T, et al. ERS/ ESTS/EACTS/ESTRO guidelines for the management of malignant pleural mesothelioma. Eur Respir J 2020;55:1900953.

31. Boutin C, Rey F. Thoracoscopy in pleural malignant mesothelioma: a prospective study of 188 consecutive patients. Part 1: Diagnosis. Cancer 1993;72:389-93.

32. Edmondstone WM. Investigation of pleural effusion: comparison between fibreoptic thoracoscopy, needle biopsy and cytology. Respir Med 1990;84:23-6.

33. Righi L, Duregon E, Vatrano S, et al. BRCA1-Associated Protein 1 (BAP1) Immunohistochemical Expression as a Diagnostic Tool in Malignant Pleural Mesothelioma Classification: A Large Retrospective Study. J Thorac Oncol 2016;11:2006-17.

34. Ordóñez NG. Application of immunohistochemistry in the diagnosis of epithelioid mesothelioma: a review and update. Hum Pathol 2013;44:1-19.

35. Husain AN, Colby T, Ordonez N, et al. Guidelines for pathologic diagnosis of malignant mesothelioma: 2012 update of the consensus statement from the International Mesothelioma Interest Group. Arch Pathol Lab Med 2013;137:647-67.

36. Wan C, Shen YC, Liu MQ, et al. Diagnostic value of fluorescence in situ hybridization assay in malignant mesothelioma: a meta-analysis. Thailand; 2012.

37. by IASLC 2016. Staging Manual in Thoracic Oncology.: 2nd Edition PART III PLEURAL MESOTHELIOMA Page 135-153. Available online: https://www.iaslc.org/Portals/0/staging_handbook_2016_ hi-res_restricted_use_only_per_iaslc_permission. pdf?ver=2019-05-08-202520-383

38. Byrne MJ, Nowak AK. Modified RECIST criteria for assessment of response in malignant pleural mesothelioma. Ann Oncol 2004;15:257-60.

39. Plathow C, Klopp M, Thieke C, et al. Therapy response in malignant pleural mesothelioma-role of MRI using RECIST, modified RECIST and volumetric approaches in comparison with CT. Eur Radiol 2008;18:1635-43.

40. Tsao AS, Garland L, Redman M, et al. A practical guide of the Southwest Oncology Group to measure malignant pleural mesothelioma tumors by RECIST and modified RECIST criteria. J Thorac Oncol 2011;6:598-601.

41. Armato SG, Oxnard GR, MacMahon H, et al. Measurement of mesothelioma on thoracic CT scans: a comparison of manual and computer-assisted techniques. Med Phys 2004;31:1105-15.

42. Sharif S, Zahid I, Routledge T, et al. Does positron emission tomography offer prognostic information in malignant pleural mesothelioma? Interact Cardiovasc Thorac Surg 2011;12:806-11.

43. Zahid I, Sharif S, Routledge T, et al. What is the best way to diagnose and stage malignant pleural mesothelioma? Interact Cardiovasc Thorac Surg 2011;12:254-9.

44. Plathow C, Staab A, Schmaehl A, et al. Computed tomography, positron emission tomography, positron emission tomography/computed tomography, and magnetic resonance imaging for staging of limited pleural mesothelioma: initial results. Invest Radiol 2008;43:737-44.

45. Rice DC, Steliga MA, Stewart J, et al. Endoscopic ultrasound-guided fine needle aspiration for staging of malignant pleural mesothelioma. Ann Thorac Surg 2009;88:862-8; discussion 868-9.

46. Alvarez JM, Hasani A, Segal A, et al. Bilateral thoracoscopy, mediastinoscopy and laparoscopy, in addition to CT, MRI and PET imaging, are essential to correctly stage and treat patients with mesothelioma prior to trimodality therapy. ANZ J Surg 2009;79:734-8.

47. Rice DC, Erasmus JJ, Stevens CW, et al. Extended Surgical Staging for Potentially Resectable Malignant Pleural Mesothelioma. Ann Thorac Surg 2005;80:1988-92; discussion 1992-3.

48. Opitz I, Friess M, Kestenholz P, et al. A New Prognostic Score Supporting Treatment Allocation for Multimodality Therapy for Malignant Pleural Mesothelioma: A Review of 12 Years' Experience. J Thorac Oncol 2015;10:1634-41.

49. de Perrot M, Dong Z, Bradbury P, et al. Impact of tumour thickness on survival after radical radiation and surgery in malignant pleural mesothelioma. Eur Respir J 2017;49:1601428.

50. Ricciardi S, Cardillo G, Zirafa CC, et al. Surgery for malignant pleural mesothelioma: an international guidelines review. J Thorac Dis 2018;10:S285-92.

51. Taioli E, Wolf AS, Camacho-Rivera M, et al. Determinants of Survival in Malignant Pleural Mesothelioma: A Surveillance, Epidemiology, and End Results (SEER) Study of 14,228 Patients. PLoS One 2015;10:e0145039. 
52. Hoda MA, Klikovits T, Arns M, et al. Management of malignant pleural mesothelioma-part 2: therapeutic approaches : Consensus of the Austrian Mesothelioma Interest Group (AMIG). Wien Klin Wochenschr 2016;128:618-26.

53. Sugarbaker DJ, Jaklitsch MT, Bueno R, et al. Prevention, early detection, and management of complications after 328 consecutive extrapleural pneumonectomies. J Thorac Cardiovasc Surg 2004;128:138-46.

54. Rintoul RC, Ritchie AJ, Edwards JG, et al. Efficacy and cost of video-assisted thoracoscopic partial pleurectomy versus talc pleurodesis in patients with malignant pleural mesothelioma (MesoVATS): an open-label, randomised, controlled trial. Lancet 2014;384:1118-27.

55. Treasure T, Lang-Lazdunski L, Waller D, et al. Extrapleural pneumonectomy versus no extra-pleural pneumonectomy for patients with malignant pleural mesothelioma: clinical outcomes of the Mesothelioma and Radical Surgery (MARS) randomised feasibility study. Lancet Oncol 2011;12:763-72.

56. Warnock C, Lord K, Taylor B, et al. Patient experiences of participation in a radical thoracic surgical trial: findings from the Mesothelioma and Radical Surgery Trial 2 (MARS 2). Trials 2019;20:598.

57. Taioli E, Wolf AS, Flores RM. Meta-analysis of survival after pleurectomy decortication versus extrapleural pneumonectomy in mesothelioma. Ann Thorac Surg 2015;99:472-80.

58. Magouliotis DE, Tasiopoulou VS, Athanassiadi K. Updated meta-analysis of survival after extrapleural pneumonectomy versus pleurectomy/decortication in mesothelioma. Gen Thorac Cardiovasc Surg 2019;67:312-20.

59. Flores RM, Pass HI, Seshan VE, et al. Extrapleural pneumonectomy versus pleurectomy/decortication in the surgical management of malignant pleural mesothelioma: results in 663 patients. J Thorac Cardiovasc Surg 2008;135:620-6, 626.e1-3.

60. Cao C, Tian D, Park J, Allan J, Pataky KA, Yan TD. A systematic review and meta-analysis of surgical treatments for malignant pleural mesothelioma. Lung Cancer 2014;83:240-5.

61. Schwartz RM, Lieberman-Cribbin W, Wolf A, et al. Systematic review of quality of life following pleurectomy decortication and extrapleural pneumonectomy for malignant pleural mesothelioma. BMC Cancer 2018;18:1188.

62. Lauk O, Hoda MA, Perrot M de, et al. Extrapleural pneumonectomy after induction chemotherapy: perioperative outcome in 251 mesothelioma patients from three high-volume institutions. Ann Thorac Surg 2014;98:1748-54.

63. Sugarbaker DJ, Richards WG, Bueno R. Extrapleural pneumonectomy in the treatment of epithelioid malignant pleural mesothelioma: novel prognostic implications of combined N1 and N2 nodal involvement based on experience in 529 patients. Ann Surg 2014;260:577-80; discussion 580-2.

64. Perrot M de, Feld R, Cho BCJ, et al. Trimodality therapy with induction chemotherapy followed by extrapleural pneumonectomy and adjuvant high-dose hemithoracic radiation for malignant pleural mesothelioma. J Clin Oncol 2009;27:1413-8.

65. Matthews C, Freeman C, Sharples LD, et al. MesoTRAP: a feasibility study that includes a pilot clinical trial comparing video-assisted thoracoscopic partial pleurectomy decortication with indwelling pleural catheter in patients with trapped lung due to malignant pleural mesothelioma designed to address recruitment and randomisation uncertainties and sample size requirements for a phase III trial. BMJ Open Respir Res 2019;6:e000368.

66. Opitz I, Lauk O, Meerang M, et al. Intracavitary cisplatinfibrin chemotherapy after surgery for malignant pleural mesothelioma: A phase I trial. J Thorac Cardiovasc Surg 2019. [Epub ahead of print]. doi: 10.1016/ j.jtcvs.2019.07.073.

67. Vogelzang NJ, Rusthoven JJ, Symanowski J, et al. Phase III study of pemetrexed in combination with cisplatin versus cisplatin alone in patients with malignant pleural mesothelioma. J Clin Oncol 2003;21:2636-44.

68. Arnold DT, Hooper CE, Morley A, et al. The effect of chemotherapy on health-related quality of life in mesothelioma: results from the SWAMP trial. Br J Cancer 2015;112:1183-9.

69. Zalcman G, Mazieres J, Margery J, et al. Bevacizumab for newly diagnosed pleural mesothelioma in the Mesothelioma Avastin Cisplatin Pemetrexed Study (MAPS): a randomised, controlled, open-label, phase 3 trial. Lancet 2016;387:1405-14.

70. Krug LM, Pass HI, Rusch VW, et al. Multicenter phase II trial of neoadjuvant pemetrexed plus cisplatin followed by extrapleural pneumonectomy and radiation for malignant pleural mesothelioma. J Clin Oncol 2009;27:3007-13.

71. van Meerbeeck JP, Gaafar R, Manegold C, et al. Randomized phase III study of cisplatin with or without raltitrexed in patients with malignant pleural 
mesothelioma: an intergroup study of the European Organisation for Research and Treatment of Cancer Lung Cancer Group and the National Cancer Institute of Canada. J Clin Oncol 2005;23:6881-9.

72. Bottomley A, Gaafar R, Manegold C, et al. Short-term treatment-related symptoms and quality of life: results from an international randomized phase III study of cisplatin with or without raltitrexed in patients with malignant pleural mesothelioma: an EORTC LungCancer Group and National Cancer Institute, Canada, Intergroup Study. J Clin Oncol 2006;24:1435-42.

73. Bearz A, Talamini R, Rossoni G, et al. Re-challenge with pemetrexed in advanced mesothelioma: a multiinstitutional experience. BMC Res Notes 2012;5:482.

74. Ceresoli GL, Zucali PA, Mencoboni M, et al. Phase II study of pemetrexed and carboplatin plus bevacizumab as first-line therapy in malignant pleural mesothelioma. Br J Cancer 2013;109:552-8.

75. Zucali PA, Perrino M, Lorenzi E, et al. Vinorelbine in pemetrexed-pretreated patients with malignant pleural mesothelioma. Lung Cancer 2014;84:265-70.

76. O'Brien MER, Watkins D, Ryan C, et al. A randomised trial in malignant mesothelioma $(\mathrm{M})$ of early (E) versus delayed (D) chemotherapy in symptomatically stable patients: the MED trial. Ann Oncol 2006;17:270-5.

77. Yajnik S, Rosenzweig KE, Mychalczak B, et al. Hemithoracic radiation after extrapleural pneumonectomy for malignant pleural mesothelioma. Int J Radiat Oncol Biol Phys 2003;56:1319-26.

78. Rusch VW, Rosenzweig K, Venkatraman E, et al. A phase II trial of surgical resection and adjuvant high-dose hemithoracic radiation for malignant pleural mesothelioma. J Thorac Cardiovasc Surg 2001;122:788-95.

79. Krayenbuehl J, Oertel S, Davis JB, et al. Combined photon and electron three-dimensional conformal versus intensity-modulated radiotherapy with integrated boost for adjuvant treatment of malignant pleural mesothelioma after pleuropneumonectomy. Int J Radiat Oncol Biol Phys 2007;69:1593-9.

80. Patel PR, Yoo S, Broadwater G, et al. Effect of increasing experience on dosimetric and clinical outcomes in the management of malignant pleural mesothelioma with intensity-modulated radiation therapy. Int J Radiat Oncol Biol Phys 2012;83:362-8.

81. Gomez DR, Hong DS, Allen PK, et al. Patterns of failure, toxicity, and survival after extrapleural pneumonectomy and hemithoracic intensity-modulated radiation therapy for malignant pleural mesothelioma. J Thorac Oncol 2013;8:238-45.

82. Thieke C, Nicolay NH, Sterzing F, et al. Long-term results in malignant pleural mesothelioma treated with neoadjuvant chemotherapy, extrapleural pneumonectomy and intensity-modulated radiotherapy. Radiat Oncol 2015;10:267.

83. Bille A, Belcher E, Raubenheimer H, et al. Induction chemotherapy, extrapleural pneumonectomy, and adjuvant radiotherapy for malignant pleural mesothelioma: experience of Guy's and St Thomas' hospitals. Gen Thorac Cardiovasc Surg 2012;60:289-96.

84. Allen AM, Czerminska M, Jänne PA, et al. Fatal pneumonitis associated with intensity-modulated radiation therapy for mesothelioma. Int J Radiat Oncol Biol Phys 2006;65:640-5.

85. Rice DC, Smythe WR, Liao Z, et al. Dose-dependent pulmonary toxicity after postoperative intensity-modulated radiotherapy for malignant pleural mesothelioma. Int J Radiat Oncol Biol Phys 2007;69:350-7.

86. Rosenzweig KE, Zauderer MG, Laser B, et al. Pleural intensity-modulated radiotherapy for malignant pleural mesothelioma. Int J Radiat Oncol Biol Phys 2012;83:1278-83.

87. Chance WW, Rice DC, Allen PK, et al. Hemithoracic intensity modulated radiation therapy after pleurectomy/ decortication for malignant pleural mesothelioma: toxicity, patterns of failure, and a matched survival analysis. Int J Radiat Oncol Biol Phys 2015;91:149-56.

88. Stewart SA, Clive AO, Maskell NA, et al. Evaluating quality of life and cost implications of prophylactic radiotherapy in mesothelioma: Health economic analysis of the SMART trial. PLoS One 2018;13:e0190257.

89. Bayman N, Appel W, Ashcroft L, et al. Prophylactic Irradiation of Tracts in Patients With Malignant Pleural Mesothelioma: An Open-Label, Multicenter, Phase III Randomized Trial. J Clin Oncol 2019;37:1200-8.

90. Boutin C, Rey F, Viallat JR. Prevention of malignant seeding after invasive diagnostic procedures in patients with pleural mesothelioma. A randomized trial of local radiotherapy. Chest 1995;108:754-8.

91. O'Rourke N, Garcia JC, Paul J, et al. A randomised controlled trial of intervention site radiotherapy in malignant pleural mesothelioma. Radiother Oncol 2007;84:18-22.

92. Clive AO, Taylor H, Dobson L, et al. Prophylactic radiotherapy for the prevention of procedure-tract metastases after surgical and large-bore pleural procedures 
in malignant pleural mesothelioma (SMART): a multicentre, open-label, phase 3, randomised controlled trial. Lancet Oncol 2016;17:1094-104.

93. MacLeod N, Chalmers A, O'Rourke N, et al. Is Radiotherapy Useful for Treating Pain in Mesothelioma?
A Phase II Trial. J Thorac Oncol 2015;10:944-50.

94. World Health Organization. Elimination of asbestos related diseases. Available online: https://www.who.int/ipcs/ assessment/public_health/Elimination_asbestos-related_ diseases_EN.pdf

Cite this article as: Schumann SO, Kocher G, Minervini F. Epidemiology, diagnosis and treatment of the malignant pleural mesothelioma, a narrative review of literature. J Thorac Dis 2021;13(4):2510-2523. doi: 10.21037/jtd-20-2761 